Harvey Rosen, Department of Mathematics, University of Alabama, Tuscaloosa, AL 35487

\title{
ON CHARACTERIZING EXTENDABLE CONNECTIVITY FUNCTIONS BY ASSOCIATED SETS
}

\begin{abstract}
We answer two questions in [11]. We show that the class of extendable connectivity functions from $I$ into $I(I=[0,1])$ cannot be characterized in terms of associated sets, and we show that one of Jones' functions obeying $f(x+y)=f(x)+f(y)$ is an example of an almost continuous function from $\mathbb{R}$ into $\mathbb{R}$ which is not the uniform limit of any sequence of extendable connectivity functions.
\end{abstract}

A class $K$ of real-valued functions defined on an interval is characterized by associated sets if there exists a family $P$ of subsets of $\mathbb{R}$ such that $f \in K$ if and only if for every $\alpha \in \mathbb{R}$, the "associated" sets $E^{\alpha}(f)=\{x: f(x)<\alpha\}$ and $E_{\alpha}(f)=\{x: f(x)>\alpha\}$ belong to $P$. For example, the family $P$ of open sets characterizes the class $K$ of continuous functions.

A Darboux function $f: I \rightarrow I$ has the intermediate value property. A function $f: I \rightarrow I$ has the Weak Cantor Intermediate Value Property (WCIVP) if for each subinterval $(x, y)$ of $I$ with $f(x) \neq f(y)$, there exists a Cantor set $C$ in $(x, y)$ such that $f(C)$ lies between $f(x)$ and $f(y)$. A connectivity function $f: I \rightarrow I$ or $F: I^{2} \rightarrow I$ obeys the property that the graph of its restriction to each connected subset of its domain is a connected set. A connectivity function $f: I \rightarrow I$ is extendable if there exists a connectivity function $F: I^{2} \rightarrow I$ such that $F(x, 0)=f(x)$ for all $x \in I$. Each neighborhood of the graph of an almost continuous function $f: I \rightarrow I$ in $I \times I$ contains the graph of a continuous function from $I$ into $I$. Similar definitions hold for $\mathbb{R}$ replacing $I$.

Of the above classes of functions, these have been shown by the following to be not characterizable by associated sets:

Key Words: extendable connectivity function, associated set, almost continuous function, uniform limit

Mathematical Reviews subject classification: 26A15, 54C08

Received by the editors February, 29, 1996 

Darboux functions
Bruckner [1]
connectivity functions
Cristian and Tevy [3]
almost continuous functions
Kellum [9]

We continue this pattern for extendable connectivity functions.

Theorem 1 The class $K$ of extendable connectivity functions from I into I cannot be characterized by associated sets.

Proof. Assume $K$ is characterized by a family $P$ of associated sets. It follows from [12] or [2] that there exists an extendable connectivity function $f: I \rightarrow I$ whose graph is dense in $I^{2}$ and $f(I)=(0,1)$. By [11], there exists a dense $G_{\delta}$-subset $A$ of $(0,1)$ that is $f$-negligible. This means that every function from $I$ into $I$ obtained by arbitrarily redefining $f$ on $A$ is still an extendable connectivity function. Therefore the function $g: I \rightarrow I$ defined by

$$
g(x)= \begin{cases}f(x) & \text { if } x \in I \backslash A \\ 0 & \text { if } x \in A\end{cases}
$$

belongs to $K$, and so $E_{0}(g)=\{x \in I: g(x)>0\}=I \backslash A \in P$. As in [12], we show that $I \backslash A$ is negligible for some extendable connectivity function. Since $I \backslash A$ is of the first category, it follows from Lemma 3 in [10] that there exists a homeomorphism $h: I \rightarrow I$ such that $(I \backslash A) \cap h(I \backslash A)=\emptyset . I \backslash A \subset h^{-1}(A)$ and $f \circ h: I \rightarrow(0,1)$. According to Corollary 1 and Lemma 2 in [10], $f \circ h$ is an extendable connectivity function, and $h^{-1}(A)$ is $(f \circ h)$-negligible. Therefore $I \backslash A$ is $(f \circ h)$-negligible. Then the function $\phi: I \rightarrow I$ defined by

$$
\phi(x)= \begin{cases}(f \circ h)(x) & \text { if } x \in A \\ 0 & \text { if } x \in I \backslash A\end{cases}
$$

belongs to $K$, and so $E_{0}(\phi)=\{x \in I: \phi(x)>0\}=A \in P$. Define the function $\psi: I \rightarrow I$ by

$$
\psi(x)= \begin{cases}0 & \text { if } x \in A \\ 1 & \text { if } x \in I \backslash A .\end{cases}
$$

Then for every $\alpha \in \mathbb{R}, E^{\alpha}(\psi), E_{\alpha}(\psi) \in P$, yet $\psi \notin K$, a contradiction.

Theorem 2 The class $K$ of uniform limits of sequences of extendable connectivity functions $f_{n}: \mathbb{R} \rightarrow \mathbb{R}$ is not characterized by associated sets. 
Proof. Assume $K$ is characterizable in terms of a family $P$ of associated sets. By [12] or [2], there exists an extendable connectivity function $g: \mathbb{R} \rightarrow \mathbb{R}$ whose graph is dense in $\mathbb{R}^{2}$. According to Theorem 1 in [11] which still holds there for $\mathbb{R}$ replacing $I$, there exists a dense $G_{\delta}$-subset $A$ of $\mathbb{R}$ that is $g$-negligible. We may suppose $A \cap g^{-1}(1 / 2)=\emptyset$. Therefore $g^{-1}(1 / 2)=\cup_{i=1}^{\infty} C_{i}$, where each $C_{i}$ is nowhere dense in $\mathbb{R}$ and if $i \neq j$, then $C_{i} \cap C_{j}=\emptyset$. Like Example 1 in [11], for each positive integer $n$, define $f_{n}: \mathbb{R} \rightarrow \mathbb{R}$ by

$$
f_{n}(x)= \begin{cases}g(x) & \text { if } x \in \mathbb{R} \backslash \cup_{i=1}^{n} C_{i} \\ \frac{i+2}{2 i} & \text { if } x \in C_{i} \text { and } i=1,2, \ldots, n\end{cases}
$$

Then the sequence of extendable connectivity functions $f_{n}$ converges uniformly to a function $f: \mathbb{R} \rightarrow \mathbb{R}$ with range $\mathbb{R} \backslash\{1 / 2\}$. Then $f \in K$ and so $E^{1 / 2}(f)$, $E_{1 / 2}(f) \in P$. Define a function $h: \mathbb{R} \rightarrow \mathbb{R}$ by

$$
h(x)= \begin{cases}1 & \text { if } x \in E^{1 / 2}(f) \\ 0 & \text { if } x \in E_{1 / 2}(f)\end{cases}
$$

Clearly, $h$ cannot be a uniform limit of a sequence of Darboux functions, and so $h \notin K$. Yet for every $\alpha \in \mathbb{R}, E^{\alpha}(h), E_{\alpha}(h) \in P$, and so $h \in K$, a contradiction.

In [5], Gibson and Roush gave an example of a Darboux function $f: I \rightarrow \mathbb{R}$ which is not the uniform limit of a sequence of connectivity functions, and in [6], Jastrzebski gave an example of a connectivity function $f: I \rightarrow \mathbb{R}$ which is not the uniform limit of a sequence of almost continuous functions. We continue this trend with an example of an almost continuous function $f: \mathbb{R} \rightarrow$ $\mathbb{R}$ that is not the uniform limit of any sequence of extendable connectivity functions. We prove two preliminary results.

Theorem 3 There exists a function $f: \mathbb{R} \rightarrow \mathbb{R}$ obeying $f(x+y)=f(x)+f(y)$ that is almost continuous but does not have the WCIVP.

Proof. Jones constructed a function $f: \mathbb{R} \rightarrow \mathbb{R}$ such that $f(x+y)=$ $f(x)+f(y)$ and such that its graph intersects every perfect subset $P$ of $\mathbb{R}^{2}$ with $x$-projection $\pi_{1}(P)$ having $c$-many points [7]. Kellum showed it was almost continuous [8].

Suppose $x<y, f(x) \neq f(y)$, and $C$ is an arbitrary Cantor set between $x$ and $y$. By construction, $f$ meets the perfect subset $C \times\{f(x)\}$ of $\mathbb{R}^{2}$, and so $f(C)$ does not lie between $f(x)$ and $f(y)$. Therefore $f$ does not have the $W C I V P$. 
According to [4], an extendable connectivity function has the WCIVP. Therefore the example given by Theorem 3 is not an extendable connectivity function.

Theorem 4 The uniform limit $f$ of a sequence of extendable connectivity functions $f_{n}: \mathbb{R} \rightarrow \mathbb{R}$ has the WCIVP.

Proof. Suppose $x<y$ and $f(x) \neq f(y)$. Choose $x_{1}$ and $y_{1}$ such that $x<x_{1}<y_{1}<y, f\left(x_{1}\right) \neq f\left(y_{1}\right)$, and $f\left(x_{1}\right)$ and $f\left(y_{1}\right)$ lie between $f(x)$ and $f(y)$. We may suppose $f(x)<f\left(x_{1}\right)<f\left(y_{1}\right)<f(y)$. Let

$$
\epsilon=(1 / 2) \min \left\{f\left(x_{1}\right)-f(x), f(y)-f\left(y_{1}\right), f\left(y_{1}\right)-f\left(x_{1}\right)\right\} .
$$

There is an integer $N$ such that for every $n \geq N$ and for every $z \in \mathbb{R}, \mid f(z)-$ $f_{n}(z) \mid<\epsilon$. Since $f_{N}\left(x_{1}\right) \neq f_{N}\left(y_{1}\right)$, there exists a Cantor set $C \subset\left(x_{1}, y_{1}\right)$ such that $f_{N}(C) \subset\left(f_{N}\left(x_{1}\right), f_{N}\left(y_{1}\right)\right)$. Then $C \subset(x, y)$ and $f(C) \subset(f(x), f(y))$. This shows $f$ has the $W C I V P$.

Since Jones' function $f$ in Theorem 3 does not have the WCIVP, then according to Theorem $4, f$ cannot be the uniform limit of a sequence of extendable connectivity functions. This proves the following result.

Theorem 5 There exists an almost continuous function $f: \mathbb{R} \rightarrow \mathbb{R}$ that is not the uniform limit of a sequence of extendable connectivity functions from $\mathbb{R}$ into $\mathbb{R}$.

\section{References}

[1] A. M. Bruckner, On characterizing classes of functions in terms of associated sets, Canad. Math. Bull. 10 (1967), 227-231.

[2] K. Ciesielski and I. Reclaw, Cardinal invariants concerning extendable and peripherally continuous functions, Real Analysis Exch., 21, no. 2 (1995-96), 459-472

[3] B. Cristian and I. Tevy, On characterizing connected functions, Real Anal. Exchange 6 (1980-81), 203-207.

[4] R. G. Gibson and F. Roush, Concerning the extension of connectivity functions, Top. Proc. 10 (1985), 75-82.

[5] R. G. Gibson and F. Roush, The uniform limit of connectivity functions, Real Anal. Exchange 11 (1985-86), no. 1, 254-259. 
[6] J. Jastrzebski, An answer to a question of R. G. Gibson and F. Roush, Real. Anal. Exchange 15 (1989-90), 340-341.

[7] F. B. Jones, Connected and disconnected plane sets and the functional equation $f(x)+f(y)=f(x+y)$, Bull. Amer. Math. Soc. 48 (1942), $115-120$.

[8] K. R. Kellum, Sums and limits of almost continuous functions, Colloq. Math. 31 (1974), 125-128.

[9] K. R. Kellum, Almost continuity and connectivity - sometimes it's as easy to prove a stronger result, Real Anal. Exchange 8 (1982-1983), no. 1, $244-252$.

[10] T. Natkaniec, Extendability and almost continuity, Real Anal. Exchange 21 (1995-96), no. 1, 349-355.

[11] H. Rosen, Limits and sums of extendable connectivity functions, Real Anal. Exchange 20 (1994-95), no. 1, 183-191.

[12] H. Rosen, Every real function is the sum of two extendable connectivity functions, Real Anal. Exchange 21 (1995-96), no. 1, 299-303. 